\title{
A Dexamethasone-regulated Gene Signature Is Prognostic for Poor Survival in Glioblastoma Patients
}

\author{
Markus M. Luedi, MD, MBA, *† Sanjay K. Singh, PhD, Jennifer C. Mosley, MS, $\dagger$ \\ Masumeh Hatami, MD, $\dagger$ Joy Gumin, BS,§ Erik P. Sulman, MD, PhD, // Frederick F. Lang, MD, $\S$ \\ Frank Stueber, MD,* Pascal O. Zinn, MD, PhD, $₫ \#$ and Rivka R. Colen, MD**
}

\begin{abstract}
Background: Dexamethasone is reported to induce both tumorsuppressive and tumor-promoting effects. The purpose of this study was to identify the genomic impact of dexamethasone in glioblastoma stem cell (GSC) lines and its prognostic value; furthermore, to identify drugs that can counter these side effects of dexamethasone exposure.
\end{abstract}

Methods: We utilized 3 independent GSC lines with tumorigenic potential for this study. Whole-genome expression profiling and pathway analyses were done with dexamethasone-exposed and control cells. GSCs were also co-exposed to dexamethasone and temozolomide. Risk scores were calculated for most affected genes, and their associations with survival in The Cancer Genome Atlas and Repository of Molecular Brain Neoplasia Data databases. In silico Connectivity Map analysis identified camptothecin as antagonist to dexamethasone-induced negative effects.

Received for publication April 16, 2016; accepted August 3, 2016. From the *Department of Anesthesiology, Bern University Hospital Inselspital, Bern, Switzerland; Departments of †Cancer Systems Imaging; \$Diagnostic Imaging; §Neurosurgery and Brain Tumor Center; \|Radiation Oncology, Division of Radiation Oncology; \#Neurosurgery, Cancer Systems Imaging, and Cancer Biology; **Cancer Systems Imaging, and Diagnostic Imaging, The University of Texas MD Anderson Cancer Center; and Department of Neurosurgery, Baylor College of Medicine, Houston, TX.

Supported by Mach-Gaensslen Foundation, Unteraegeri, Switzerland. Alfred \& Anneliese Sutter-Stoettner Foundation, Muenchwilen, Switzerland. National Institue of Health (NIH), grant 1P50CA12CA127001, Bethesda, MD. John S. Dunn Sr. Distinguished Chair in Diagnostic Imaging, Houston TX. The University of Texas MD Anderson Cancer Center Start-up Fund, Houston TX.

M.M.L., S.K.S., J.C.M., M.H., P.O.Z., and R.R.C: helped design the study, conduct the study, analyze the data, and write the manuscript. J.G., E.P.S. and F.F.L.: helped design the study and conduct the study. F.S.: helped design the study, analyze the data, and write the manuscript.

The authors have no conflicts of interest to disclose.

Address correspondence to: Rivka R. Colen, MD, Department of Cancer Systems Imaging. The University of Texas MD Anderson Cancer Center, 1881 East Road, 3SCR4, Houston, TX 77030 (e-mail: rcolen@mdanderson.org).

Supplemental Digital Content is available for this article. Direct URL citations appear in the printed text and are provided in the HTML and PDF versions of this article on the journal's Website, www.jnsa.com.

Copyright (C) 2016 Wolters Kluwer Health, Inc. All rights reserved.

DOI: $10.1097 /$ ANA.0000000000000368
Results: Pathway analyses predicted an activation of dexamethasone network ( $z$-score: 2.908). Top activated canonical pathways included "role of breast cancer 1 in DNA damage response" $(P=1.07 \mathrm{E}-04)$. GSCs were protected against temozolomide-induced apoptosis when coincubated with dexamethasone. Altered cellular functions included cell movement, cell survival, and apoptosis with $z$-scores of $2.815,5.137$, and -3.122 , respectively. CCAAT/enhancer binding protein beta (CEBPB) was activated in a dose dependent manner specifically in slow-dividing "stem-like" cells. CEBPB was activated in dexamethasone-treated orthotopic tumors. Patients with high risk scores had significantly shorter survival. Camptothecin was validated as potential partial neutralizer of dexamethasoneinduced oncogenic effects.

Conclusions: Dexamethasone exposure induces a genetic program and CEBPB expression in GSCs that adversely affects key cellular functions and response to therapeutics. High risk scores associated with these genes have negative prognostic value in patients. Our findings further suggest camptothecin as a potential neutralizer of adverse dexamethasone-mediated effects.

Key Words: dexamethasone, glioblastoma, glioblastoma stem cells, TCGA, Kaplan-Meier analysis

(J Neurosurg Anesthesiol 2016;00:000-000)

G lioblastoma is the most common malignant primary brain tumor in adults, ${ }^{1}$ with a median survival of 15.3 to 21.7 months. ${ }^{2}$ Neural, proneural, classical, and mesenchymal subtypes have been described, whereof mesenchymal glioblastoma are described as the most aggressive. $^{3}$ The transcription factor CCAAT/enhancer binding protein beta (CEBPB) is proposed to be a master regulator of mesenchymal programming in glioblastoma. ${ }^{4}$ Capillaries in glioblastoma do not evince the physiological blood-brain barrier and thus exhibit increased permeability leading to edema. ${ }^{5}$ Dexamethasone is the clinical standard for treating vasogenic edema-induced local mass effects and elevated intracranial pressure in glioblastoma. ${ }^{6}$

Although dexamethasone is effective in managing edema, its molecular impact remains controversial: it has been reported to induce proliferation in glioblastoma cell lines, ${ }^{7}$ while other studies demonstrated that it has 
inhibitory effects on proliferation of human glioblastoma cell lines in vitro. ${ }^{8}$ A rat model study generated the proposal that dexamethasone reduces both the expression and the response of vascular endothelial growth factor (VEGF), thus reducing edema. ${ }^{9}$ Dexamethasonemediated induction of apoptosis in human glioblastoma cell lines as well as its antitumor activity in xenografted nude rats have been described, ${ }^{10}$ and it has also been suggested that dexamethasone reduces invasiveness of human glioblastoma cells in vitro and blood vessel invasion in vivo through a MAPK phosphatase-1-dependent mechanism. ${ }^{11}$ Furthermore, glioma growth was reduced in mice following treatment with dexamethasone or antiangiopoietin 2.12 Recently, however, Wong and colleagues showed that glioblastoma patients who received a dexamethasone dose $>4.1 \mathrm{mg} / \mathrm{d}$ had significantly shorter OS than those who received $\leq 4.1 \mathrm{mg} / \mathrm{d}$. The study attributed this finding to immunologic interference in treatment efficacy. ${ }^{13}$ Thus, the drug's impact on biology is controversial.

Singh et $\mathrm{al}^{14}$ strongly supported the key role of the cancer stem cell hypothesis ${ }^{15}$ in glioblastoma, whereby glioblastoma stem cells (GSCs) appear to retain multiple salient features of the original tumor and contribute to therapeutic resistance and tumor recurrence. Since the publication of that report, GSCs derived from human tumors have become a reliable tool for investigating glioblastoma biology in the laboratory. So far there are no data on the impact of dexamethasone exposure on GSCs. Therefore, we aimed to identify key genes and molecular mechanisms regulated by dexamethasone in patient-derived GSC lines. We further aimed to assess the prognostic significance of a risk score based on these altered genes in glioblastoma patients and to identify clinically relevant antagonists.

\section{MATERIALS AND METHODS}

\section{Ethics Approvals}

GSC lines were derived from MD Anderson Cancer Center patients who gave written informed consent in advance and in accordance with an MD Anderson institutional review board-approved protocol (LAB040001).

All animal use complied with institutional and governmental laws and regulations and was approved by the MD Anderson institutional animal care and use committee (protocol 00001100-RN00) in accordance with the guidelines of the American Association for Laboratory Animal Science.

\section{Proliferation of Patient-derived GSCs}

To obtain a robust representation of glioblastoma (GBM), 3 independent MD Anderson patient-derived GSC lines (GSC1, GSC3, and GSC6) were isolated and cultured from surgical specimens as described elsewhere. ${ }^{14,16}$ In brief, cells were grown in GSC medium consisting of Dulbecco modified eagle medium (DMEM)/ F12 (Corning, Corning, NY) including L-glutamine
(Sigma, St Louis, MO), $1 \times$ penicillin/streptomycin (Corning), $1 \times$ B27 without vitamin A (Life Technologies, Carlsbad, CA), $20 \mathrm{ng} / \mathrm{mL}$ basic fibroblast growth factor (VWR, Radnor, PA), and $20 \mathrm{ng} / \mathrm{mL}$ epidermal growth factor (EMD Millipore, Billerica, MA) at $37^{\circ} \mathrm{C}$ in presence of $5 \% \mathrm{CO}_{2}$ as defined previously by Singh et al. ${ }^{14}$ Cells were passaged according to standard protocols with 3 minutes exposure to Accutase cell detachment solution (EMD Millipore) at $37^{\circ} \mathrm{C}$.

\section{Differentiation Assays}

GSCs were differentiated in tissue culture flasks and in chamber slides (Labtek, Scotts Valley, CA) and pretreated with poly-L-lysine (PLL) (Sigma) overnight at $37^{\circ} \mathrm{C}$. The 3 GSC lines were allowed to attach to PLL coated plates. After 12 hours, the medium was changed to differentiation medium containing DMEM, $1 \times$ penicillin/streptomycin, and $10 \%$ fetal bovine serum (Denville Scientific, Holliston, MA). The medium was exchanged every 48 hours. Cells differentiated in flasks were harvested on day 7 with 3 minutes exposure to $0.25 \%$ Trypsin solution (Corning), whereas differentiated cells in chamber slides were fixed with $4 \%$ paraformaldehyde for 10 minutes at room temperature before proceeding to immunofluorescence analysis.

\section{Flow Cytometry}

GSC lines were cultured in GSC medium for proliferation assays. After Accutase treatment, single cells were fixed with $4 \%$ paraformaldehyde for 10 minutes at room temperature and were washed twice with phosphate-buffered saline solution. Cells were permeabilized with permeabilization buffer containing $1 \%$ bovine serum albumin, $0.1 \%$ Triton $\mathrm{X}-100$, and $0.09 \%$ sodium azide for 15 minutes at room temperature. Permeabilized cells were then incubated with mouse anti-sex determining region Y-box 2 (Sox2), a stem cell marker (R\&D Systems, Minneapolis, MN); another stem cell marker, rabbit anti-nestin (NES) (Thermo Scientific Pierce, Waltham, MA); and cell proliferation marker rabbit anti-Ki67 (Thermo Scientific Pierce) in permeabilization buffer for 30 minutes at room temperature. After 3 washes with permeabilization buffer, speciesspecific allophycocyanin-conjugated secondary antibodies anti-mouse (BD Biosciences, San Jose, CA) and antirabbit (Thermo Scientific Novex, Grand Island, NY) were used for indirect labeling according to the manufacturer's protocol. Fluorescence-activated cell sorting (FACS) analysis was performed with a Gallios flow cytometer (Beckman Coulter, Brea, CA: Flow Cytometry and Cellular Imaging core facility, MD Anderson Cancer Center). The gates for sorting were based on isotype control samples for each GSC line. The FACS data were analyzed with FlowJo software (version 10).

\section{Western Blot Analysis}

Total proteins were extracted from the three independent GSC lines in the presence of protease and phosphatase inhibitors (Thermo Scientific Pierce) and 
analyzed with Western blotting for specific proteins. Briefly, each whole-cell extract (50 to $100 \mu \mathrm{g}$ ) was resolved on a $10 \%$ to $12.5 \%$ sodium dodecyl sulfate polyacrylamide gel electrophoresis (Fisher BioReagents, Pittsburgh, PA) and transferred to a nitrocellulose membrane. The membranes were probed with mouse anti-Sox2 (R\&D Systems), rabbit anti-NES (Thermo Scientific Pierce), rabbit anti-Ki67 (Thermo Scientific Pierce), and mouse anti-glyceraldehyde-3-phosphate dehydrogenase (GAPDH) (Abcam, Cambridge, MA) primary antibodies overnight at $4^{\circ} \mathrm{C}$. Rabbit anticleaved PARP (Cell Signaling), was used as apoptosis marker. The membranes were incubated with species-specific horseradish peroxidase-conjugated secondary antibodies for 1 hour at room temperature. The membranes were exposed to Clarity Western ECL Substrate (BioRad, Hercules, CA) and scanned with a c600 imaging system (Azure Biosystems, Dublin, CA). Scanned images were analyzed with cSeries Capture Software. Specific protein bands in Western blots were quantified with ImageJ software.

\section{Immunofluorescence Analysis}

Cells were differentiated for 7 days on PLL coated Lab-Tek II Chamber slides (50,000 cells per chamber), fixed as described above, and stained with mouse antiGFAP (glial fibrillary acidic protein) antibody and goat anti-mouse rhodamine-conjugated secondary antibody (both, Thermo Scientific Pierce). Nuclei were counterstained with 4',6-diamidino-2-phenylindole. Images were taken with a DFC3000 G microscope (Leica, Buffalo Grove, IL) using a $\times 20$ objective and analyzed with LAS$\mathrm{X}$ software.

\section{Orthotopic Tumor Formation}

Nine $(3 \times 3)$ male nude mice (strain nu/nu, 5 wk old; obtained from the Department of Veterinary Medicine and Surgery at MD Anderson Cancer Center) were implanted with a nylon bolt in an orthotopic position $2 \mathrm{~mm}$ posterior and $1.5 \mathrm{~mm}$ lateral to the bregma in the right cerebral hemisphere. The animals were anesthetized through this procedure, receiving ketamine intraperitoneally as described elsewhere. ${ }^{16}$ One week after bolting, each animal was injected with GSC1, GSC3, or GSC $6\left(1 \times 10^{5}\right)$ in DMEM $(5 \mu \mathrm{L})$ over 5 minutes through the bolt, again under anesthesia and with intraperitoneal ketamine. ${ }^{16}$ Mice were killed with hypercarbia and decapitation at the earliest onset of neurological symptoms and their brains were collected in formalin for paraffin embedding. Embedded tumor-bearing brains were cut into $5-\mu \mathrm{m}$ slices and deparaffinized in xylene, rehydrated in an alcohol series (100\% to $70 \%)$ and double distilled water, and stained with hematoxylin and eosin (H\&E), all according to standard protocols. H\&E-stained slides were imaged with an Olympus BX51 microscope (Olympus USA, Center Valley, PA) using a $\times 10$ objective, and the images analyzed with QCapture software.

\section{Dexamethasone, Temozolomide, and Camptothecin Treatment}

A broad range of dexamethasone concentrations have been used for in vitro studies, ${ }^{17}$ and the concentration we used $(50 \mu \mathrm{m})$ falls within this range. ${ }^{18}$ Single cell suspension of GSCs after 3 minutes exposure to Accutase cell detachment solution (EMD Millipore) were exposed for 6 days to GSC medium containing dexamethasone $50 \mu \mathrm{M}$ (Sigma) or GSC medium alone as control. Medium was exchanged daily. To validate the in silico prediction in vitro, GSC 3 and 6 were exposed to 0 , 25,50 , and $100 \mu \mathrm{m}$ dexamethasone over 3 days under stress of concomitant treatment with $500 \mu \mathrm{m}$ of the alkylating chemotherapeutic agent temozolomide (Sigma). Medium was exchanged daily. Total proteins were extracted and analyzed with Western blotting as described above. GSCs exposed to dexamethasone for 5 days were coexposed to $10 \mu \mathrm{M}$ camptothecin ${ }^{19}$ for 24 hours and cells were harvested for total RNA isolation followed by reverse transcriptase and quantitative polymerase chain reaction (PCR).

\section{Label Retention Analysis}

GSC1 and GSC3 were exposed to $5 \mu \mathrm{M}$ of CellTrace carboxyfluorescein succinimide ester Cell Proliferation substrate (Thermo Scientific Novex, Grand Island, NY) for 20 minutes at room temperature followed by removal of free dye from the solution as per the manufacturer's protocol and treated with $50 \mu \mathrm{M}$ dexamethasone (daily exchange) for 8 to 10 days. Cells underwent FACS sorting for high versus low CFSE dye retaining cells. Gates for FACS were set with negative and positive samples (CFSE stained on the day of sorting).

\section{RNA Isolation, Whole-Genome Expression Profiling, Reverse Transcriptase and Real Time PCR}

RNA was isolated with Purezol RNA Isolation Reagent (BioRad) according to the manufacturer's protocol. After quality control, RNA was analyzed with the Human Transcriptome 2.0 Microarray (Affymetrix, Santa Clara, CA) according to the producer's standards (Microarray core facility, Department of Genetics, MD Anderson Cancer Center). Probe cell intensity data (.cel format) were uploaded and normalized across all 3 GSC lines, either treated with dexamethasone or untreated (controls), using the Expression Console software (Affymetrix) with gene level analysis. All gene confidence levels (core, extended, and full) were included in the analysis, as recommended by the manufacturer. Expression profiles were analyzed with the Transcriptome Analysis Console (Affymetrix), employing a cutoff of \pm 2.0 fold-change and a normalization $z$-score of $\geq 2.0$ as described in a previous publication. ${ }^{20,21}$ In brief, the $z$ score adjusts data within a single probe. Values for individual genes are expressed as the standard deviation from the normalized mean of zero. ${ }^{22}$

The selected genes were assessed with the ingenuity pathway analysis platform (Qiagen, Valencia, CA) to 
predict dexamethasone-induced cellular functions, their networks of upstream regulators and canonical pathways. A heatmap of predicted upstream regulators and differentially expressed genes was generated with the Gene E program (www.broadinstitute.org/).

Approximately 100 to $200 \mathrm{ng}$ of total RNA were used as template in reverse transcriptase reaction with iScript cDNA synthesis kit (BioRad) as per manufacturer's protocol. The cDNAs were then amplified with SYBR Green-based PCR (BioRad) using CFX real-time system. Data were analyzed with BioRad CFX manager and $-2^{\Delta \Delta C t}$ values were plotted after normalization with GAPDH (housekeeping gene), $P$-value were calculated by 2-tailed T-Test between samples. Gene specific primers used were: GAPDH-forward: 5'AAGGTGAAGG TCGGAGTCAA3'; reverse: 5'AATGAAGGGGTCATT GATGG3'. CEBPB - forward: 5' CGTGTACACACGC GTTCAG3'; reverse: 5'CTCTCTGCTTCTCCCTCTG C3'. Sox2 - forward: 5' GCTTAGCCTCGTCGATGAAC3'; reverse: 5'AACCCCAAGATGCACAACTC3'. FKBP5forward: $5^{\prime}$ GCGGAGAGTGACGGAGTC3'; reverse: 5'T GGGGCTTTCTTCATTGTTC3'. MT1X-forward: 5' GC AAATGCAAAGAGTGCAAA3'; reverse: 5'CTTTGCA GATGCAGCCCT3'. TIMP4 - forward: 5' ACGCCTTTTG ACTCTTCCCT3'; reverse: 5'TTTCCATCACTGAGGAC CTG3'.

\section{In Vivo Model}

After tumor forming capacity of GSC3 was confirmed, we injected 10 mice with $1 \times 10^{5}$ human GSC3 as described above. Upon the day of the orthotopic injection, 5 mice in each group were treated daily with $2 \mathrm{mg} /$ $\mathrm{kg}\left(50 \mu \mathrm{g}\right.$ in $\left.100 \mu \mathrm{L} \mathrm{H}_{2} \mathrm{O}\right)$ dexamethasone (Sigma Aldrich) intraperitoneally, as described in previous publications. ${ }^{23}$ The 5 control animals received $100 \mu \mathrm{L} \quad \mathrm{H}_{2} \mathrm{O}$ intraperitoneally daily. Animals mice were killed with hypercarbia and decapitation at the earliest onset of neurological symptoms, in accordance with MD Anderson's institutional animal research protocol. Some of the brains were immediately collected in formalin for paraffin embedding and later $\mathrm{H} \& \mathrm{E}$ and immunohistochemical staining. Others were placed in collection solution and then processed with a NeuroCult enzymatic dissociation kit (Stemcell Technologies, Vancouver, BC, Canada) to obtain orthotopic tumor cells for ex vivo expression analysis.

\section{Histopathologic Staining}

Paraffin-embedded brains from the dexamethasonetreated and control mice were cut into $5-\mu \mathrm{m}$ slices, mounted on slides, deparaffinized in xylene, rehydrated in a descending alcohol series, and every 10th slide stained with H\&E according to standard protocols. To assess CEBPB protein expression in the orthotopically implanted tumors, we stained tumor samples with a CEBPB specific antibody (Abcam, San Francisco, CA), and incubated overnight at $4^{\circ} \mathrm{C}$.

The slides were then incubated with polyclonal secondary antibodies (Abcam) and exposed to peroxidase
(Abcam) and DAB substrate (Vector Laboratories, Burlingame, CA) according to the manufacturers' protocols. Counterstaining with haematoxylin was performed with standard procedures; finally, probes were pictured with an Olympus BX51 microscope (Olympus USA) and images were acquired using QCapture software.

\section{Risk Score Calculation and Clinical Outcome Analyses}

Genes with $\geq 3.5$ fold-change in expression upon dexamethasone exposure were analyzed for survival in the patient databases of The Cancer Genome Atlas (TCGA) and the Repository of Molecular Brain Neoplasia Data (REMBRANDT). Small nucleolar RNAs were excluded, as they are not present on the U133A microarray platform. Cox regression coefficient of each gene was calculated by using a Cox hazard model. A risk score (RS) was calculated for each patient by using the following equation:

$$
R S=\sum_{i=1}^{n} \beta_{i} \cdot x_{i}
$$

where $\beta_{i}$ and $x_{i}$ are the Cox regression coefficient and expression levels of the $i$ th gene of the signature. ${ }^{24}$ Using median risk score as a cutoff, patients were dichotomized into high-risk and low-risk groups. Difference in overall survival (OS) for the 2 groups was analyzed by the Kaplan-Meier (KM) method with a 2-sided log rank test. OS was defined as the time between the date of pathologic diagnosis and the date of death or the date of last clinical follow-up visit. To evaluate the effect of age and functional impairment on the prognostic effects of the gene signature, we calculated the age and Karnofsky Performance Score-adjusted hazard ratio in a multivariable Cox regression model in the TCGA cohort. A $P$-value $\leq 0.05$ was considered statistically significant. Statistical analyses were done by using R (version 3.2.2) and SPSS (version 22) software. Expression $z$-scores of individual genes (the gene expressions have been $z$-scored for each sample over the entire gene platform panel $)^{25}$ in specific molecular subgroups of GBM, that is, mesenchymal and proneural were obtained from GBM-BioDP software (http://gbmbiodp.nci.nih.gov). For single gene clinical outcome, patients were dichotomized based on median expression $z$-score ${ }^{25}$ and KM curve analysis were performed using GraphPad Prism 6 software. $P$-value is calculation is based on Gehan-Breslow-Wilcoxon test.

\section{Connectivity Map Analysis}

The genes identified as being regulated by dexamethasone in GSCs were then analyzed by the patternmatching Connectivity Map platform to identify therapeutic agents that could potentially counteract the predicted dexamethasone-induced oncogenic effects. The upregulated and downregulated genes (mRNA, foldchange $\leq 2.0$ or $\geq 2.0$ in microarray) in their rank order were uploaded to Connectivity Map (http:// www.broadinstitute.org), where a collection of genomewide expression profiles from human cell lines treated 
with bioactive agents is archived. ${ }^{26}$ The details of Connectivity Map analysis are described elsewhere. ${ }^{27,28}$ In brief, the platform creates a "connectivity score" of collected and uploaded data where upregulation yields a positive score and downregulation a negative score ( 0 if no change). Respecting means of observed results, number of available cell lines, $P$-values, specificity, percentage of non-null results, and enrichment scores, the program ranks the results. The enrichment score displays the degree to which a set is overrepresented at the extremes within a ranked list. ${ }^{29}$

\section{RESULTS}

\section{Stem Cell Properties and Orthotopic Tumor Formation are Confirmed in Human Glioblastoma-derived Cells}

First, we sought to establish that our patient-derived cell lines GSC1, GSC3, and GSC6 had stem cell features and the potential to differentiate. FACS results show that a very high percentage of cells from all 3 GSC lines expressed the stem cell markers Sox 2 and NES and the proliferation marker Ki-67 (KI67) when cultured in GSC medium (Supplemental Figure 1A i-iii, Supplemental Digital Content 1, http://links.lww.com/JNA/A36). We then exposed GSCs maintained in GSC medium to differentiation conditions. Western blot results show significantly lower protein levels of stem cell and proliferation markers under differentiation conditions than under proliferation conditions (Supplemental Figure 1B, Supplemental Digital Content 1, http://links.lww.com/JNA/A36). Immunofluorescence analysis using antibody against differentiation marker GFAP show expression of the marker in all three GSC lines cultured in differentiation medium (Supplemental Figure 1Ci-iii, Supplemental Digital Content 1, http://links.lww.com/JNA/A36). All 3 GSC lines, when implanted at orthotopic locations in mouse brains, gave rise to xenograft tumors which bore hallmarks of glioblastoma, such as high cellularity, areas of necrosis, and hypervascularization (Supplemental Figure 1D i-iii, Supplemental Digital Content 1, http://links.lww.com/JNA/ A36).

\section{Genes Associated With Cell Proliferation and Movement are Most Significantly Affected by Dexamethasone Exposure in GSCs}

Having confirmed the identity and tumorigenic potential of these GSC lines, we proceeded to assess the global impact of dexamethasone at the molecular level by microarray profiling of dexamethasone-treated GSCs $(n=3)$ and their respective controls $(n=3)$. This analysis of the expression profile data identified 333 altered genes, 290 upregulated and 43 downregulated (Affymetrix $\mathrm{Hu}-$ man Transcriptome Array 2.0 Probeset IDs of dexamethasone-regulated genes in GSC1, GSC3, and GSC6 with cutoff $>2.0$ fold-change in Supplemental Table S1, Supplemental Digital Content 2, http://links. lww.com/JNA/A37). Pathway analysis of the altered genes in our dataset predicted that the dexamethasone- regulated network was activated (activation $z$-score 2.908, $P=4.23 \mathrm{E}-08$; Figs. 1A, B), providing an unbiased validation of our expression profile data. Furthermore, genes associated with this dexamethasone network alone had significant activating impact on cellular functions such as proliferation (based on 30 genes; $P=6.14 \mathrm{E}-11$ ), cell movement (based on 20 genes; $P=3.35 \mathrm{E}-09$ ), and inhibition of cell death (based on 29 genes; $P=3.03 \mathrm{E}-1$; Fig. 1B).

Network analysis of the entire data set of dexamethasone-induced genes showed significant activation of cell survival ( $z$-score 5.137, $P=1.12 \mathrm{E}-08$; Fig. 1C), decreased apoptosis $(z$-score $-3.122, \quad P=1.31 \mathrm{E}-06$; Fig. 1D), and increased movement ( $z$-score 2.815, $P=1.61 \mathrm{E}-04$; Fig. 1E). Top affected canonical pathway was "Role of Breast Cancer (BRCA)1 DNA Damage Response" ( $z$-score 1.633, $P=1.07 \mathrm{E}-04)$. The in vitro validation of in silico prediction showed a decrease in cleaved PARP with increasing concentrations of dexamethasone indicating a protective effect against DNA damage upon concomitant treatment with $500 \mu \mathrm{M}$ Temozolomide in GSC3 and GSC6 (Fig. 1F).

As an additional filter to predict cellular functions most affected by dexamethasone exposure, we collated a list of regulators $(z$-score $> \pm 2.0)$ whose functional status was determined by downstream gene levels in our expression data in dexamethasone-treated versus control cells (Fig. 2A, Supplemental Tables S1, Supplemental Digital Content 2, http://links.lww.com/JNA/A37 and S2, Supplemental Digital Content 3, http://links. lww.com/JNA/A38 ). The cumulative effect of these upstream regulators and their respective downstream genes were then used to predict cellular functions by using an activation $z$-score of \pm 3.0 or more as cutoff. In this analysis, hepatocyte growth factor (HGF; activation $z$-score 3.572, $P=1.22 \mathrm{E}-04$; Fig. 2B), VEGF (activation $z$-score $3.335, \quad P=8.07 \mathrm{E}-07)$ and VEGFA (activation $z$-score 3.13, $P=6.82 \mathrm{E}-06$; Fig. 2C), and estrogen receptor 1 (ESR1; activation $z$-score 3.187, $P=3.39 \mathrm{E}-05$; Fig. 2D) were the top activated regulatory networks, and nuclear protein transcriptional regulator 1 (NUPR1; activation $z$-score $3.13, P=6.82 \mathrm{E}-06$; Fig. 2E) was the top inhibited network. All activated upstream regulators, together with their downstream target genes, were predicted to induce activation of cell movement and survival (Fig. 2B-D). The top inhibited upstream regulator (NUPR1) and its downstream targets (Fig. 2E), also predicted to induce activation of cell movement and survival. Interestingly CEBPB was predicted to be upregulated by dexamethasone exposure (activation $z$-score 1.69, $P=7.33 \mathrm{E}-043$; Supplemental Figure 2, Supplemental Digital Content 4, http://links.lww.com/JNA/ A39).

\section{CEBPB, A Master Regulator of Mesenchymal Programming in Glioblastoma is Activated by Dexamethasone}

To validate the predicted upregulation of CEBPB upon exposure to dexamethasone, we exposed GSC1 
A

\begin{tabular}{|c|c|c|c|c|c|}
\hline Genes & Network Status & Fold Change & & 2 & 3 \\
\hline ADAM10 & Activated & 2.78 & + & & + \\
\hline ATP1A1 & Activated & 2.18 & & + & \\
\hline BCL6 & Activated & 2.27 & + & + & \\
\hline BRCA2 & Activated & 4.61 & + & + & + \\
\hline EGFR & Activated & 2.03 & + & + & + \\
\hline FKBP5 & Activated & 7.47 & + & + & \\
\hline FN1 & Activated & 2.07 & + & & + \\
\hline GDF15 & Activated & -2.44 & + & + & + \\
\hline GJA1 & Activated & 2.2 & + & + & + \\
\hline IER3 & Activated & -2.67 & + & + & \\
\hline IFITM3 & Activated & -2.42 & + & & + \\
\hline IRS2 & Activated & 2.27 & + & + & + \\
\hline LAMC1 & Activated & 3.05 & + & & + \\
\hline MT1X & Activated & 3.97 & & + & \\
\hline PIK3R1 & Activated & 2.26 & + & + & + \\
\hline PLD1 & Activated & 2.2 & + & + & + \\
\hline PPARGC1A & Activated & 2.06 & + & + & \\
\hline PRKAA2 & Activated & 5.87 & & + & + \\
\hline SERPINA3 & Activated & 2.18 & + & + & + \\
\hline SPARCL1 & Activated & 2 & + & & + \\
\hline TIMP4 & Activated & 3.55 & + & + & \\
\hline TJP1 & Activated & 2.46 & + & & + \\
\hline CHI3L.1 & Inhibited & -2 & + & + & 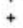 \\
\hline $\mathrm{FT}$. & Inhibited & -3.43 & + & & \\
\hline HSPA5 & Inhibited & 2.11 & + & + & + \\
\hline IGFBP5 & Inhibited & 2.24 & + & + & + \\
\hline ITGA2 & Inhibited & 2.88 & + & + & + \\
\hline SPARC & Inhibited & 2.41 & + & & + \\
\hline $\mathrm{B} 2 \mathrm{M}$ & Affected & 2.88 & + & + & \\
\hline CCND2 & Affected & 2.27 & + & + & \\
\hline KIF14 & Affected & 2.05 & & + & + \\
\hline MTHFD1 & Affected & 2.11 & + & & \\
\hline NUF2 & Affected & 2.59 & & + & \\
\hline SKA3 & Affected & 2.33 & & 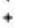 & \\
\hline STLL & Affected & 2.59 & + & + & \\
\hline TOP2A & Affected & 2.13 & + & + & \\
\hline
\end{tabular}
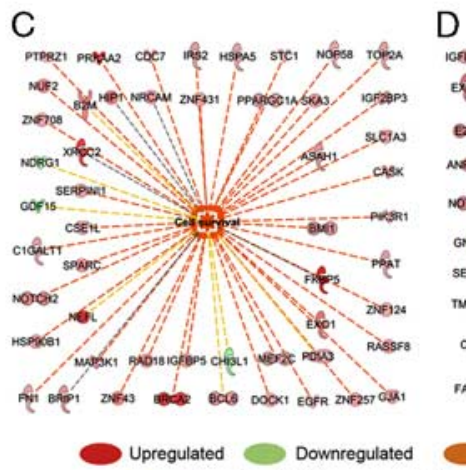

\section{D}

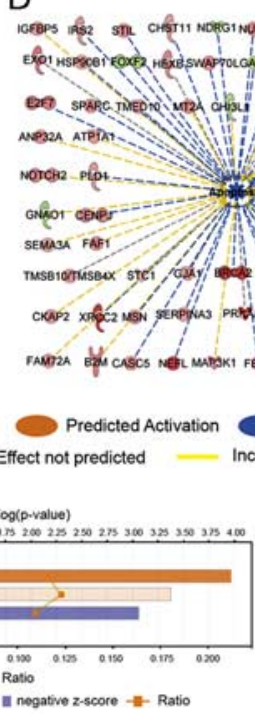

ii

$\mathrm{Fi}$
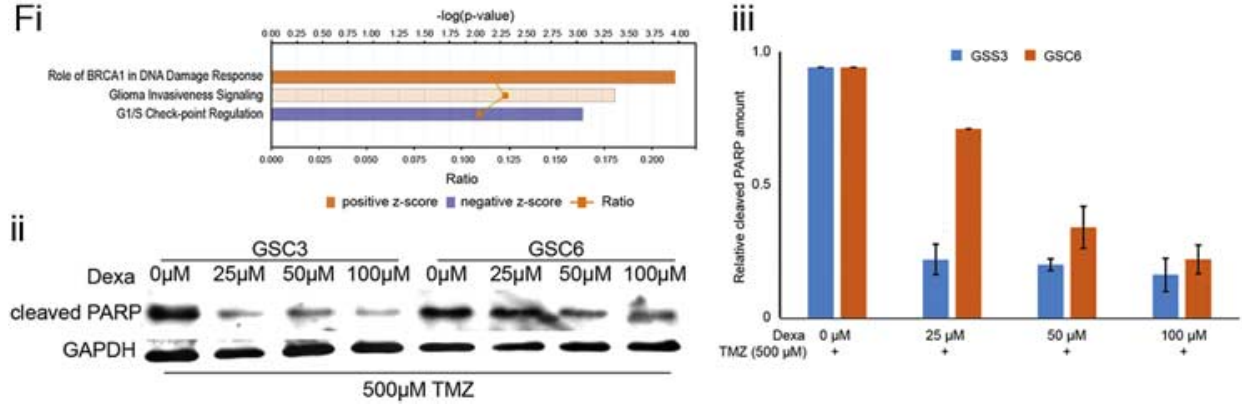

FIGURE 1. Apoptosis is inhibited and survival is activated upon dexamethasone exposure. A, Fold-changes of gene expression in the dexamethasone network predicted its activation status. Their respective associations $(+)$ with the cellular functions (1) proliferation, (2) cell death, and (3) cell movement are shown. B, This schematic representation shows the dexamethasone network, the genes involved in its activation, and its predicted impact on cellular functions. Numbers under the gene names indicate the fold-change in expression. C-E, Individual genes contributing to the activated state of cell survival (C), the inhibited state of apoptosis (D), and the activated state of cell movement (E) in GSCs in response to dexamethasone are represented schematically. Numbers under the gene names indicate the fold-change in expression. Genes and cellular functions are labeled and color coded. A color index is shown at the bottom. F, Network analysis for canonical pathway affected by dexamethasone shows "role of BRCA1 in DNA damage response" with z-score 1.633 as top result (i). Scans of Western blots of whole-cell extracts of GSC3 and GSC6 treated with different concentrations of dexamethasone over 3 days under DNA damage stress of concomitant treatment with $500 \mu \mathrm{M}$ Temozolomide and probed with an antibody against cleaved PARP as marker of apoptosis (GAPDH is loading control) (ii). Quantification of relative cleaved PARP amounts in Western blots (shown in ii) normalized with GAPDH (iii). BRAC1 indicates breast cancer 1; GAPDH, glyceraldehyde-3-phosphate dehydrogenase; GSC, glioblastoma stem cell.

Copyright (C) 2016 Wolters Kluwer Health, Inc. Unauthorized reproduction of this article is prohibited. This paper can be cited using the date of access and the unique DOI number which can be found in the footnotes 


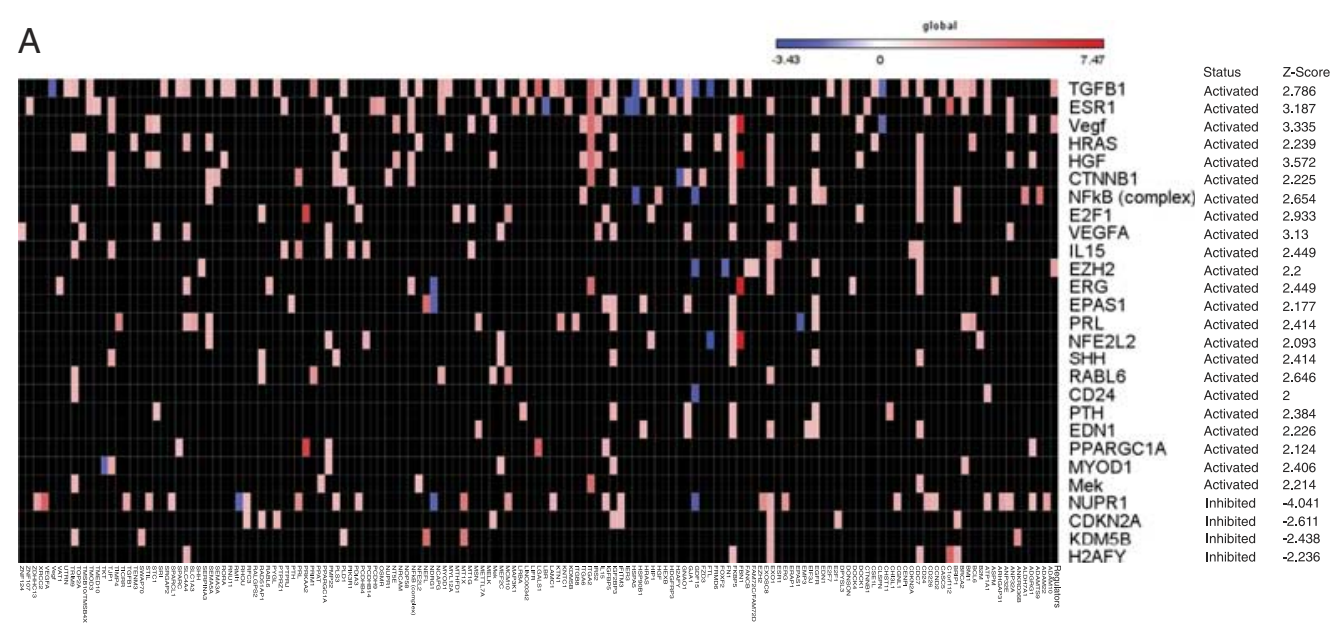

B

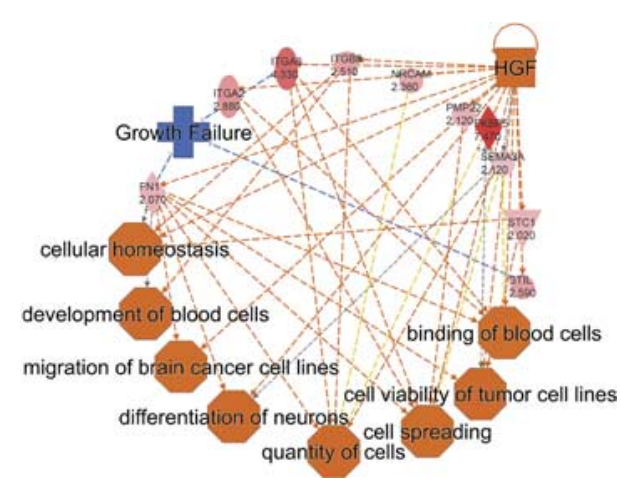

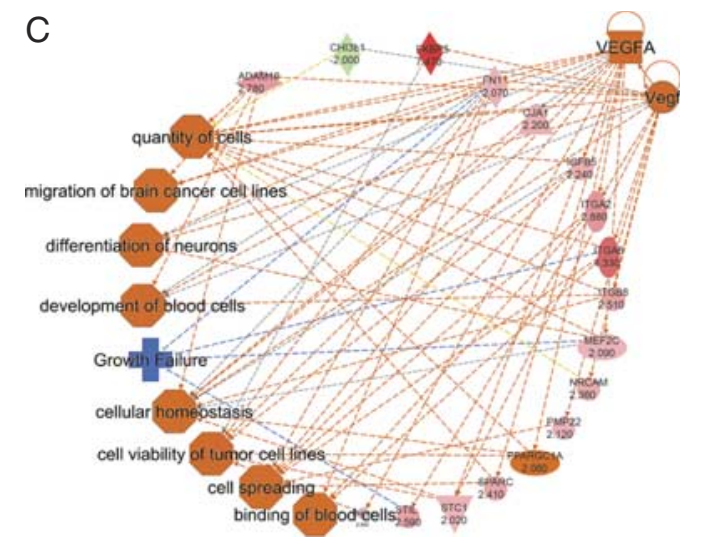

C

E

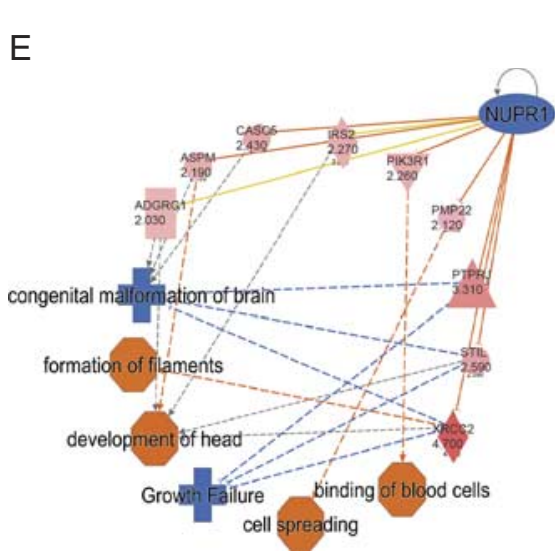

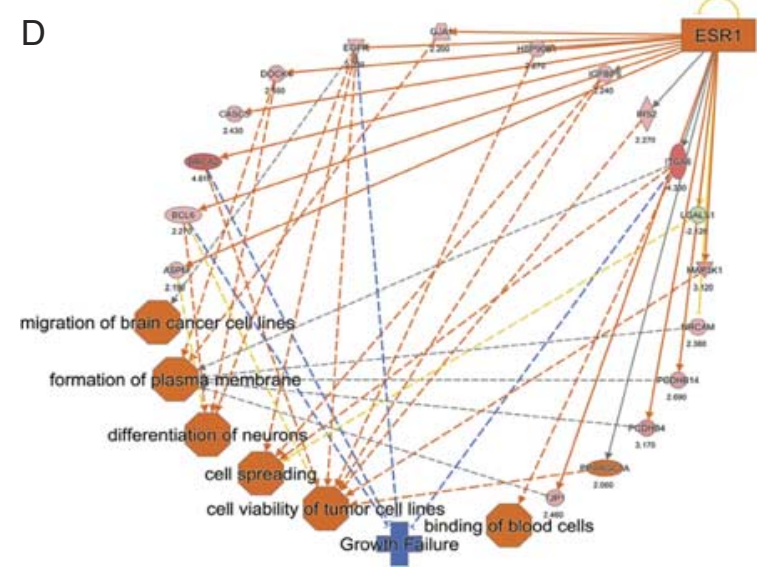

Upregulated $\bigcirc$ Downregulated $\bigcirc$ Predicted Activation $\bigcirc$ Predicted Inhibition — Leads to Activation

_Leads to Inhibition __ Inconsistent with state of downstream molecule __ Effect not predicted

FIGURE 2. Oncogenic networks are activated by dexamethasone. A, Heatmap shows genes and their association with upstream regulators with activation status $z$-scores $\geq 2.0$. The color intensity bar above the heatmap represents the range of fold-change of gene expression: red as upregulation and blue as downregulation. B, Schematic of the HGF network (activation Z-score 3.572) with the genes associated with this top activated upstream regulator and their biological implications. C, Schematic of the VEGF (activation z-score 3.335) and VEGFA (activation z-score 3.31) networks with the genes associated with this top activated upstream regulator and their biological implications. D, Schematic of the ESR1 network (activation z-score 3.187) with the genes associated with this top activated upstream regulator and their biological implications. E, Schematic of the NUPR1 network (activation z-score -4.041 ) with the genes associated with this top activated upstream regulator and their biologic implications. Genes and cellular functions are labeled and color coded. A color index is shown at the bottom. ESR1 indicates estrogen receptor 1; HGF, hepatocyte growth factor; NUPR1, nuclear protein transcriptional regulator 1; VEGFA, vascular endothelial growth factor A.

Copyright (C) 2016 Wolters Kluwer Health, Inc. Unauthorized reproduction of this article is prohibited. This paper can be cited using the date of access and the unique DOI number which can be found in the footnotes. 
A

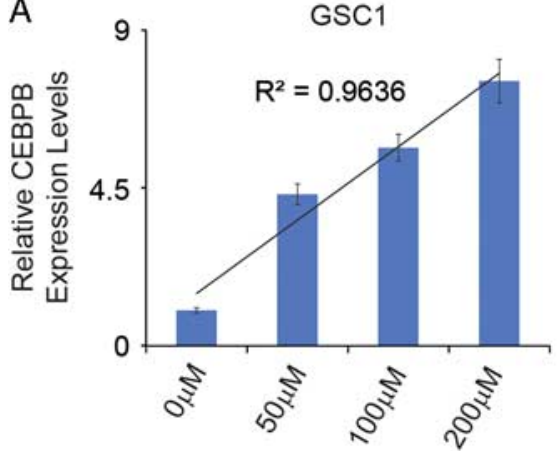

Dexamethasone
B

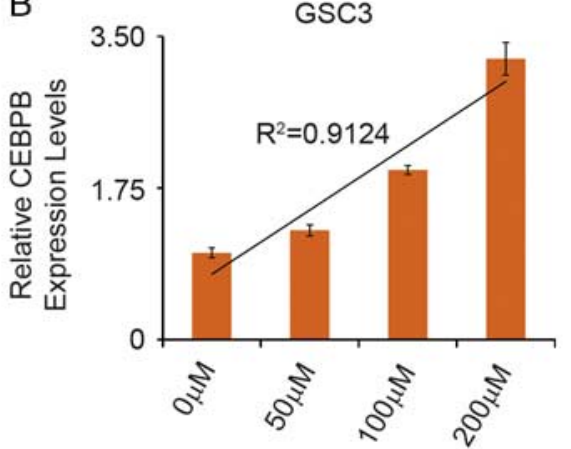

Dexamethasone
C

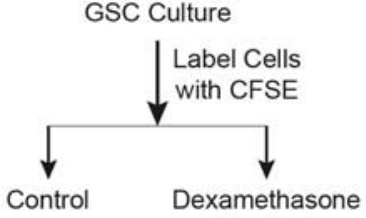
Treated

Culture 8-10 Days

FACS

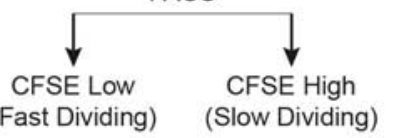

D

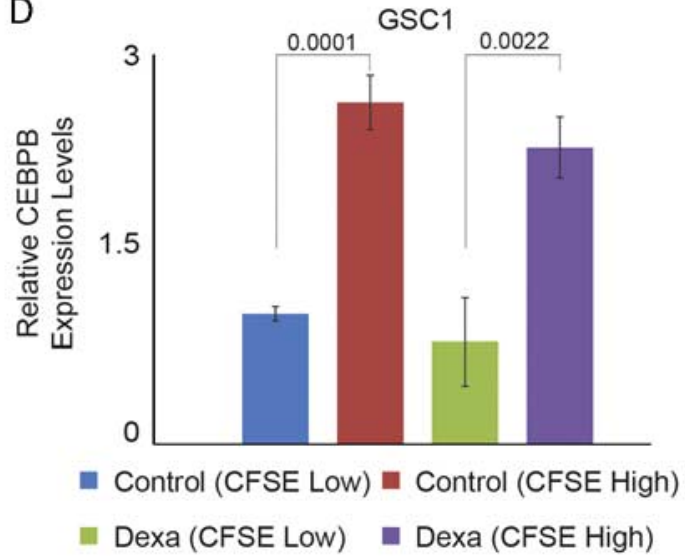

$\mathrm{F}$

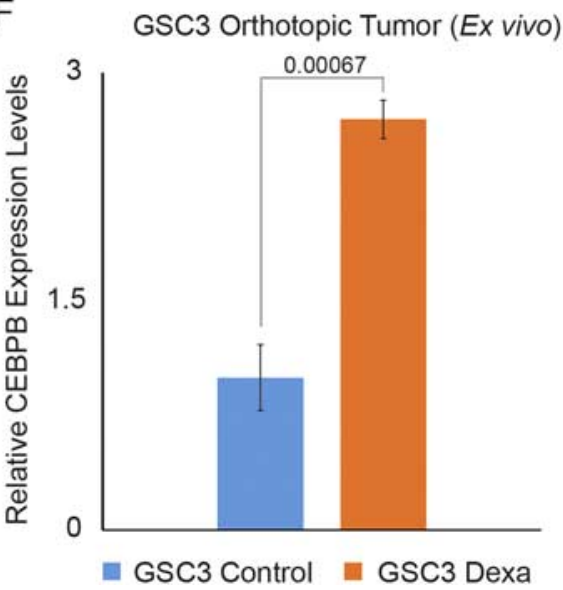

$\mathrm{E}$

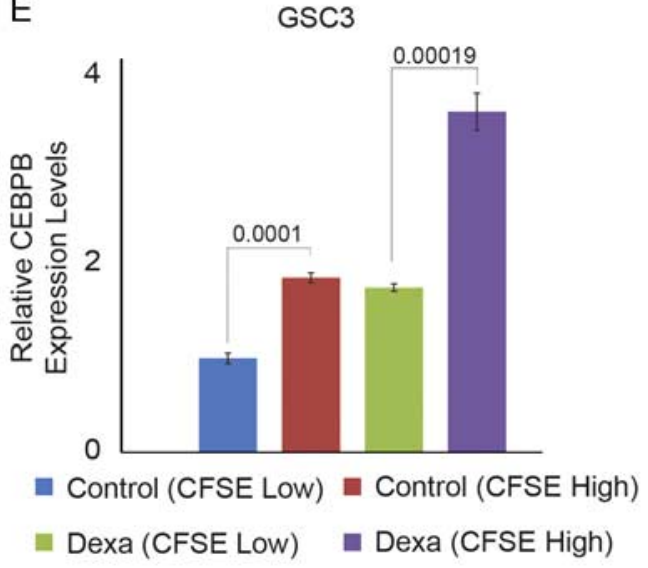

G

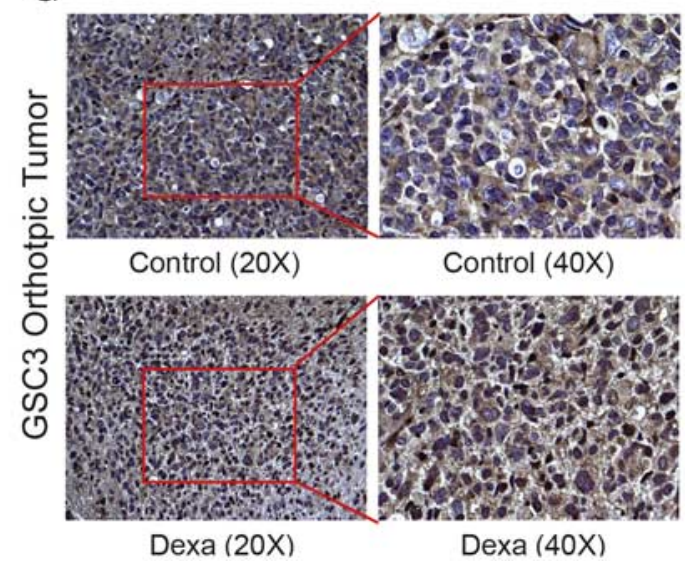

FIGURE 3. The transcription factor C/EBPbeta (CEBPB), a master regulator of the most malignant subtype of glioblastoma is activated upon dexamethasone exposure in vitro, ex vivo, and in vivo and dexamethasone treated thus CEBPB expressing cells slower division rate as indicator for cell "stemness." A, Relative expression levels of CEBPB in GSC1 upon exposure to 50, 100, and $200 \mu \mathrm{M}$ dexamethasone for 6 days. B, Relative expression levels of CEBPB in GSC3 upon exposure to 50, 100, and $200 \mu \mathrm{M}$ dexamethasone for 6 days. C, Schematic of the experiment with CFSE labeling to determine cell division rates as a readout of stemness properties of GSCs upon 8 to 10 days exposure to $50 \mu \mathrm{M}$ dexamethasone. D, CFSE labeled GSC1 cells treated with $50 \mu \mathrm{M}$ dexamethasone over 8 to 10 days selected for fast-dividing and slow-dividing subpopulations show a significant upregulation of CEBPB in slow-dividing cells upon exposure to $50 \mu \mathrm{M}$ dexamethasone over 8 to 10 days. E, CFSE labeled GSC1 cells treated with $50 \mu \mathrm{M}$ dexamethasone over 8 to 10 days selected for fast-dividing and slow-dividing subpopulations show a significant upregulation of CEBPB in slow-dividing cells upon exposure to $50 \mu \mathrm{M}$ dexamethasone over 8 to 10 days. F, Expression level analysis of GSC3 ex vivo derived orthotopic tumor cells shows a significant increase of CEBPB expression levels upon exposure of tumor bearing mice to $2 \mathrm{mg} / \mathrm{kg}$ dexamethasone daily for 4 weeks. G, immunohistochemistry staining with specific antibodies against CEBPB confirms the increase of CEBPB expression upon exposure of tumor bearing mice to $2 \mathrm{mg} / \mathrm{kg}$ dexamethasone. Red box: Area of $40 \mathrm{x}$ magnification. Brown nuclei are positive for CEBPB. P-values are displayed above the compared samples in each graph. GSC indicates glioblastoma stem cell.

Copyright (C) 2016 Wolters Kluwer Health, Inc. Unauthorized reproduction of this article is prohibited. This paper can be cited using the date of access and the unique DOI number which can be found in the footnotes. 
TABLE 1. Genes With >3.5 Fold-Change in TCGA and REMBRANDT GBM Patient Cohorts

\begin{tabular}{lccc}
\hline Gene & $\begin{array}{c}\text { Fold- } \\
\text { change }\end{array}$ & $\begin{array}{c}\text { Beta-coefficient } \\
\text { Score TCGA }\end{array}$ & $\begin{array}{c}\text { Beta-coefficient Score } \\
\text { REMBRANDT }\end{array}$ \\
\hline FKBP5 & 7.47 & 0.097 & 0.038 \\
PRKAA2 & 5.87 & -0.086 & -0.395 \\
PCDHB8 & 5.86 & 0.001 & 0.247 \\
ABCA5 & 4.84 & 0.218 & 0.188 \\
XRCC2 & 4.70 & 0.185 & -0.043 \\
BRCA2 & 4.61 & 0.056 & 0.044 \\
LIFR & 4.57 & -0.167 & -0.198 \\
LRRTM2 & 4.44 & 0.137 & 0.285 \\
ITGA6 & 4.33 & 0.098 & 0.208 \\
NEFL & 4.33 & 0.021 & -0.085 \\
MT1X & 3.97 & 0.041 & 0.311 \\
PCDHB12 & 3.61 & 0.03 & 0.066 \\
ALDH7A1 & 3.59 & -0.056 & 0.1 \\
TIMP4 & 3.55 & -0.007 & -0.129 \\
CHD7 & 3.52 & -0.204 & -0.13 \\
\hline
\end{tabular}

TCGA indicates The Cancer Genome Atlas; REMBRANDT, Repository of Molecular Brain Neoplasia Data.

and GSC 3 cells to varying doses of dexamethasone for 6 days. Our results from quantitative real time PCR show a dose dependent increase in CEBPB levels with $R^{2}$ values of 0.96 and 0.91 in GSC1 and GSC3, respectively (Figs. 3A, B). To further elucidate impact of dexamethasone on CEBPB levels in specific cell populations within GSC cultures, namely CFSE high (slow-dividing "stem-like" cells, express high levels of Sox2 (data not shown)) and label low (fast dividing "non-stem-like" cells), GSC1 and GSC3 cells were labeled with CFSE dye and treated with $50 \mu \mathrm{M}$ dexamethasone over 8 to 10 days and sorted for low (fast dividing) and high (slow dividing) subpopulations (Fig. 3C). Real time PCR data show endogenous levels of CEBPB is higher in CFSE high cells, a trend which was maintained upon dexamethasone exposure (Figs. 3D, E). CEBPB expression levels in ex vivo isolated tumor cells showed a significant upregulation upon treatment of mice with $2 \mathrm{mg} / \mathrm{kg}$ dexamethasone over 4 weeks (Fig. 3F). Immunohistochemistry staining against CEBPB protein confirmed this increase in tumor bearing sections of dexamethasone-treated mice brains (Fig. 3G).

\section{Patients With High Risk Scores for Dexamethasone-induced Genes have Poorer Prognosis and Dexamethasone-induced Upregulation of Genes Included in the Score Confirmed}

Applying a most stringent cutoff of $>3.5$ foldchange identified 15 genes upregulated by dexamethasone in GSCs (Table 1). To assess the clinical translatability of these genes, we calculated the risk scores for each gene as described in Methods. These scores were then applied to discovery (TCGA, $\mathrm{N}=515$ ) and validation (REMBRANDT, $\mathrm{N}=178$ ) data sets to identify each of these glioblastoma patients as either high or low risk. Strikingly, Kaplan-Meier analyses showed a significant survival difference between high-risk and low-risk groups (Fig. 4). Median survival durations for patients with high versus low risk score were 12.8 versus 16.7 months in the TCGA cohort $(\mathrm{N}=515, P<0.001)$ and 13.1 months versus 17.0 months in the REMBRANDT cohort $(\mathrm{N}=178, P=0.015$; Figs. 4A, B). The multivariate Cox regression analysis of the TCGA cohort (hazard ratio, $1.92 ; 95 \%$ [confidence interval] CI, 1.26-2.93; $P=0.002$ ) showed that our risk score is a valid predictor of survival independent of age (hazard ratio, 1.02; 95\% CI, 1.01-1.03; $P<0.001)$ and Karnofsky Performance Score (hazard ratio, $0.98 ; 95 \% \mathrm{CI}, 0.97-0.99 ; P<0.001)$. The dexamethasone dependence of FKBP5, MT1X, and TIMP4, 3 representative genes predicted in the 2 databases, are validated by real time PCR in vitro in GSC1 (Fig. 4C) and GSC3 (Fig. 4D).

\section{Identification and Validation of Camptothecin as Neutralizer of Dexamethasone-induced Genes In Vitro}

The dexamethasone-regulated genes with expression fold-change $\leq-2.0$ or $\geq 2.0$ were assessed with Connectivity Map, an archive of genome-wide expression profiles from human cell lines treated with bioactive agents; this archive can be used to identify interactions between these agents and genes. This analysis was undertaken to identify therapeutics that could potentially counteract adverse gene expression changes induced by dexamethasone. We identified therapeutics with enrichment scores up to -0.992 ; the top-ranked compound was camptothecin (Fig. 5A, Supplemental Table S3, Supplemental Digital Content 5, http://links.lww.com/JNA/ A40). The enrichment score reflects the degree to which a set is overrepresented at the extremes within a ranked list. To test potential antagonistic properties of camptothecin to dexamethasone-induce alterations, we exposed GSC1 and GSC3 to camptothecin for 24 hours (a time point at which we did not detect significant amount of cell death. Supplemental Figure 3, Supplemental Digital Content 6, http://links.lww.com/JNA/A41) after these cells were preexposed to dexamethasone for 5 days. Real time PCR data show a significant decrease in FKBP5 and MT1X levels in cells co-exposed to camptothecin and dexamethasone as compared with cells treated with dexamethasone alone (Figs. 5B, C). Further analysis of TCGA expression data show that FKBP5 and MT1X genes are highly expressed in the mesenchymal subgroup (Figs. 5D, F). When the proneural subgroup of patients were further subclassified into high and low groups, the high group of patients has significantly poorer clinical outcome (Figs. 5E, G).

\section{DISCUSSION}

This preclinical study demonstrated that dexamethasone altered genes predicted to promote oncogenic pathways in GSCs. High risk scores associated with genes most responsive to dexamethasone exposure were then found to be prognostic for poor outcome in 2 
A

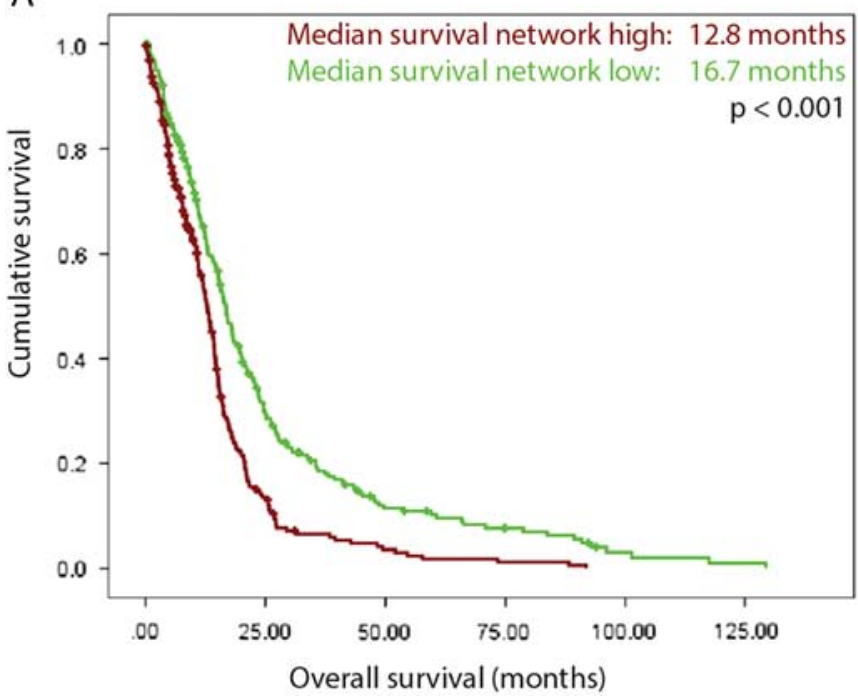

B

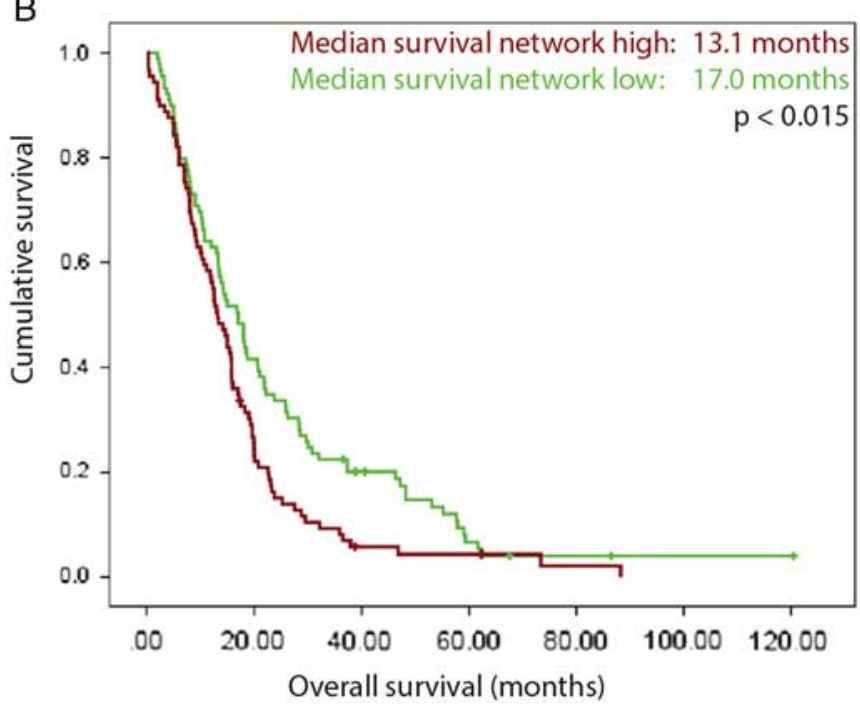

C

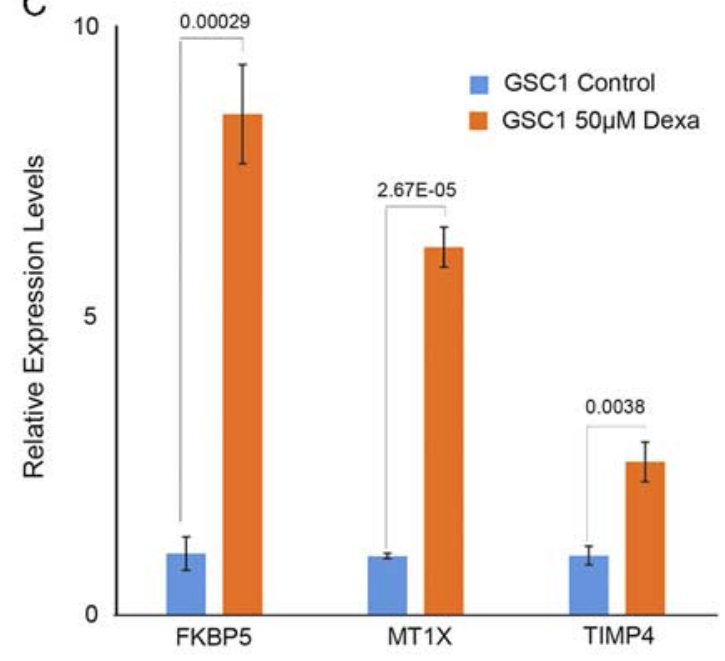

D

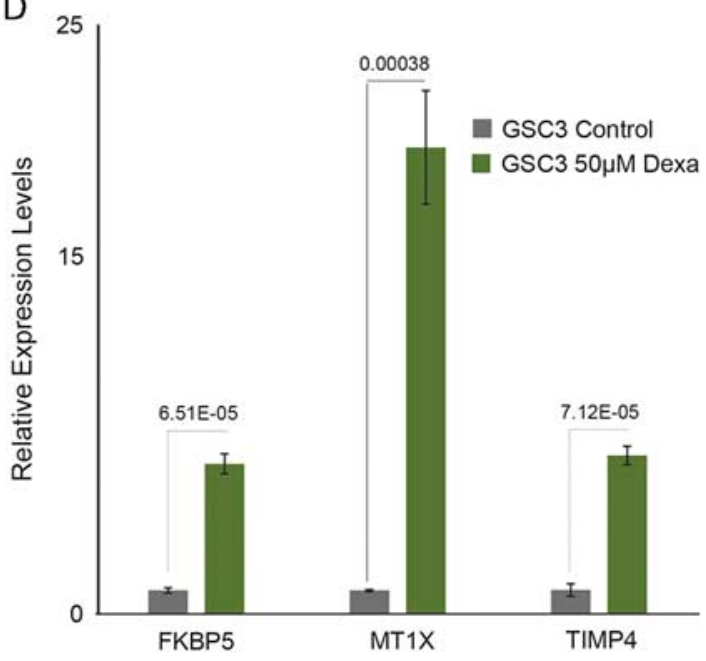

FIGURE 4. Glioblastoma patients with high risk score for dexamethasone-induced genes have poorer prognosis. A, Kaplan-Meier curve analysis of the TCGA glioblastoma cohort $(n=515)$. Median survival of glioblastoma patients with a high risk score (red) was 12.8 months, whereas that of patients with a low risk score (green) was 16.7 months $(P<0.001)$. B, Kaplan-Meier curve analysis of the REMBRANDT glioblastoma cohort $(n=178)$. Median survival of glioblastoma patients with a high risk score (red) was 13.1 months, whereas that of patients with a low risk score (green) was 17.0 months $(P=0.015)$. C and $D$, Real time PCR for FKBP5, MT1X, and TIMP4, members of dexamethasone-induced gene signature, in GSC1 and GSC3 with and without dexamethasone exposure. P-values are displayed above the compared samples in each graph. GSC indicates glioblastoma stem cell; PCR, polymerase chain reaction; REMBRANDT, Repository of Molecular Brain Neoplasia Data; TCGA, The Cancer Genome Atlas.

independent clinico-genomic glioblastoma cohorts. This is potentially of clinical importance, as dexamethasone is widely used in glioblastoma patients to treat edema and associated increased intracranial pressure during the perioperative period. Further, analysis of altered genes with Connectivity Map identified United States Food and Drug Administration (FDA) approved drugs that can counter these adverse dexamethasone-induced effects while potentially preserving the beneficial antiedema effects.
Upstream regulatory networks identified by this work are well known in the pathogenesis of glioblastoma. HGF has been shown to be involved in epithelial-to-mesenchymal transition through binding to the receptor tyrosine kinase c-MET, not only in embryogenesis but also in invasiveness of glioblastoma, because of its potent induction of mesenchymal transition in epithelium-driven cells. ${ }^{30} \mathrm{~A}$ recent clinical trial (phase II) investigated the antitumor activity of an antiHGF antibody: though no significant advantage could be

Copyright (C) 2016 Wolters Kluwer Health, Inc. Unauthorized reproduction of this article is prohibited. This paper can be cited using the date of access and the unique DOI number which can be found in the footnotes. 
A

\begin{tabular}{|c|c|c|c|c|c|c|c|}
\hline \multicolumn{7}{|c|}{ Top 10 therapeutics predicted by CMAP to inhibit dexamethasone-induced effects } & \multirow[b]{2}{*}{$\%$ non-null } \\
\hline Rank & Cmap name & Mean & No. of cell lines & Enrichment score & $P$ value & Specificity & \\
\hline 1 & Camptothecin & -0.761 & 3 & -0.992 & 0 & 0.0455 & 100 \\
\hline 2 & GW-8510 & -0.643 & 4 & -0.959 & 0 & 0.0611 & 100 \\
\hline 3 & $0175029-0000$ & -0.711 & 6 & -0.959 & 0 & 0 & 100 \\
\hline 4 & Daunorubicin & -0.622 & 4 & -0.912 & 0.0001 & 0.0088 & 100 \\
\hline 5 & Irinotecan & -0.688 & 3 & -0.938 & 0.00032 & 0.0544 & 100 \\
\hline 6 & Thioguanosine & -0.568 & 4 & -0.846 & 0.00101 & 0.0141 & 100 \\
\hline 7 & Cloperastine & -0.486 & 6 & -0.7 & 0.00169 & 0.0252 & 100 \\
\hline 8 & Ouabain & -0.564 & 4 & -0.826 & 0.00177 & 0.0351 & 100 \\
\hline 9 & Roxithromycin & -0.568 & 4 & -0.824 & 0.00181 & 0.0222 & 100 \\
\hline 10 & Clioquinol & -0.515 & 5 & -0.746 & 0.00202 & 0 & 80 \\
\hline
\end{tabular}

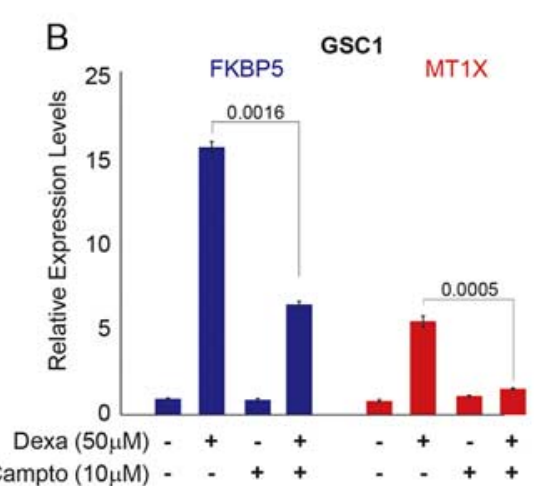

C
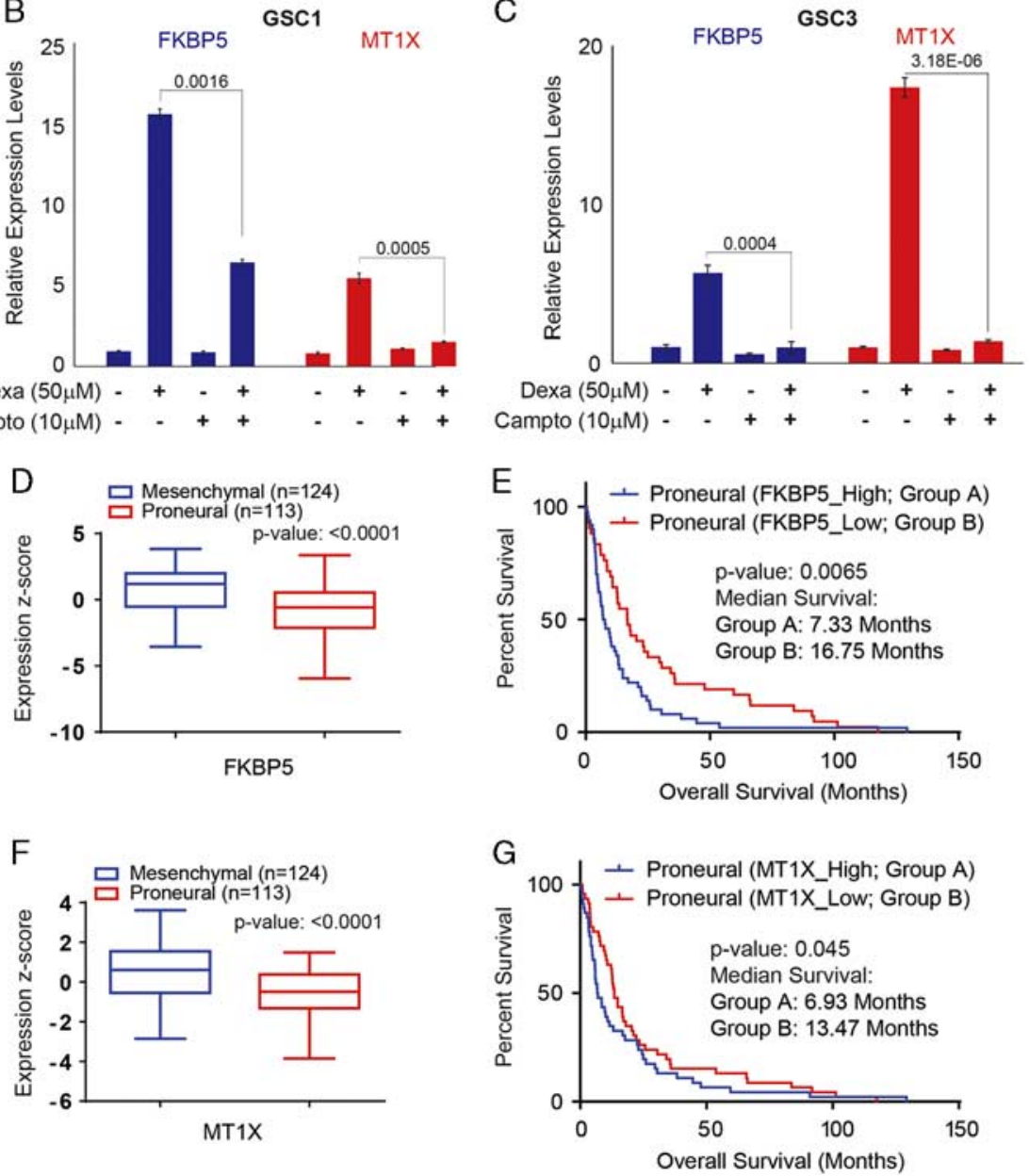

FIGURE 5. Identification and validation of camptothecin as neutralizer of dexamethasone-induced effects in GSC. A, A list of top 10 therapeutics identified by connectivity map analysis based on dexamethasone-induced genomic profiles. The ranking was automatically calculated, respecting mean observed values, number of cell lines available in the database, enrichment score, $P$-value, specificity, and percentage of non-null results. B and C, In vitro exposure of GSCs with $10 \mu \mathrm{M}$ camptothecin and $50 \mu \mathrm{M}$ dexamethasone (alone and in combinations), relative expression levels of FKBP5 and MT1X, 2 representative genes of the dexamethasone-induced oncogenic gene network in GSC1 and GSC3. P-values are displayed above the compared samples in each graph. D and F, Expression of FKBP5 and MT1X in mesenchymal and proneural subgroups of GBM patients in TCGA database. E and G, Kaplan-Meier curve analysis of the proneural TCGA glioblastoma cohort $(n=113)$. E, Median survival of proneural glioblastoma patients with a high FKBP5 expression is 7.33 months, whereas that of patients with a low FKBP5 expression is 16.75 months $(P=0.0065)$. G, Median survival of proneural glioblastoma patients with a high MT1X expression is 6.93 months, whereas that of patients with a low MT1X expression is 13.47 months $(P=0.045)$. GSC indicates glioblastoma stem cell; TCGA, The Cancer Genome Atlas.

Copyright (C) 2016 Wolters Kluwer Health, Inc. Unauthorized reproduction of this article is prohibited. This paper can be cited using the date of access and the unique DOI number which can be found in the footnotes. 
shown in patients with recurrent glioblastoma, ${ }^{31}$ HGF's contribution to glioblastoma malignancy is unquestioned. As a response to tissue hypoxia, the hypoxia-inducible factor 1 acts as a transcription factor, controlling the expression of VEGF. ${ }^{32,33}$ Both VEGF and hypoxia-inducible factor $1 \alpha$ have been shown to significantly promote both angiogenesis and invasion in glioblastoma. ${ }^{34}$ VEGFA, 1 of 5 VEGF subtypes, has been shown to be a prognostic indicator for brain tumor patients. ${ }^{35}$ Given that vascular proliferation is a key component of glioblastoma biology and that VEGF expression levels directly correlate with tumor malignancy and hence inversely with prognosis, the anti-VEGF antibody bevacizumab has been approved by the FDA for treatment of recurrent glioblastoma. ${ }^{36,37}$ The role of estrogen receptors (ESRs) in glioblastoma remains controversial: some evidence shows that ESRs are decreased in high-grade gliomas, while ESR expression levels correlated negatively with tumor differentiation. ${ }^{38}$ The ESR antagonist tamoxifen was shown to induce cell death in glioma cells ${ }^{39}$ and has been proposed as a potential chemotherapeutic agent in temozolomide-resistant glioblastoma ${ }^{40}$ Our results provide a potential molecular basis for this indication of tamoxifen, especially given that most patients with recurrent glioblastoma are further exposed to dexamethasone. NUPR1, the most inhibited dexamethasone-dependent upstream regulator we found, is involved in a variety of stress-related functions in noncancer conditions $^{41}$ and as a tumor suppressor in prostate cancer. ${ }^{42}$ Our discovery of its potential involvement in glioblastoma is novel. The dose dependent increase in CEBPB (both in vitro and in vivo) upon dexamethasone exposure, a known regulator of mesenchymal program in GBM, is indicative of a potential mesenchymal shift in tumor cells (specifically in stem-like cells), which may contribute to poorer outcome of patients.

In our screen, the cellular functions most affected by dexamethasone were activation of cellular survival and migration as well as inhibition of cellular apoptosis, all functions well known to drive glioblastoma pathogenesis. ${ }^{43,44}$ DNA repair associated genes such as the tumor suppressor BRCA1 are known to be increased upon treatment with temozolomide. ${ }^{45}$ Interestingly, the activation of BRCA1 DNA damage response was the top affected canonical pathway in our screen. The functional readout of dexamethasone and temozolomide cotreatment, however, showed a decrease of apoptosis with increasing concentrations of dexamethasone. The upstream regulatory networks contributing to these changes are known to contribute to glioblastoma malignancy. These preclinical findings are of importance as they offer a possible explanation for recent findings by Wong et $\mathrm{al}^{13}$ that glioblastoma patients who received higher dexamethasone doses had significantly shorter OS than those who received lower doses. Kaplan-Meier analysis of the risk scores associated with the most dexamethasoneresponsive genes in our work supports a potentially dexamethasone-dependent significant survival difference in the 2 most exhaustive clinical databases, TCGA and REMBRANDT. That this prognostic association remains significant after multiple corrections indicates the robustness of our screen and the possible distinct biological mechanism induced by dexamethasone that mediates a survival disadvantage, given that the vast majority of patients might have been under dexamethasone treatment when samples for the 2 databases were obtained. However, it appears likely that patients to whom larger doses of dexamethasone were administered had larger tumor and edema burden.

It remains unclear whether the described adverse effects of dexamethasone are due to its glucocorticoid or drug specific effect. Comparison with another corticosteroid would shed light on what is leading to the changes in gene transcription. However, the fact that cerebral edema is currently mainly treated with dexamethasone, an ethical dilemma might occur to test other steroids' effects. Dexamethasone is essential not only for acute treatment of edema in glioblastoma but also as a long-term adjuvant for chemotherapy and radiation therapy. ${ }^{46}$ Identification of the FDA-approved camptothecin by our enrichment analysis suggests a potential route for future investigations to identify the drug's potential to reduce unwanted dexamethasone effects. Our in vitro results suggest that, as predicted in silico, short-term exposure of camptothecin is able to counter dexamethasone effects at least partially. However, the presented evidence that dexamethasone increases tumor cell survival is based on gene analysis, cell survival and proliferation in vitro. To provide strong evidence that dexamethasone increases tumor cell survival, both the size of orthotopic tumors and orthotopic tumor bearing animals' survival upon dexamethasone treatment remain to be evaluated. In brief, these findings resulting from an in vitro screen warrant further investigation in an in vivo model to uncover a definitive mechanism.

In summary, our GSC data shine light on the intriguing controversy of dexamethasone-induced effects in cancer biology and provide novel insight into the potential molecular mechanisms of these effects. We demonstrate that dexamethasone induces a gene signature that is a significant predictor of poor prognosis in glioblastoma patients. It is conceivable that neutralizing dexamethasone-induced oncogenic effects, for example, with camptothecin, may become an integral part of glioblastoma therapy. The data presented here may represent a stepping stone to improving glioblastoma treatment and prolonging patient survival.

\section{ACKNOWLEDMENTS}

IRB human cells: Professor Colleen Gallagher, chief and executive director clinical ethics, The University of Texas MD Anderson Cancer Center, Houston TX.

IRB mouse work: Professor David Johnson, chair of the Institutional Animal Care and Use Committee (IACUC), The University of Texas MD Anderson Cancer Center, Houston TX. 


\section{REFERENCES}

1. Wen PY, Kesari S. Malignant gliomas in adults. $N$ Engl J Med. 2008;359:492-507.

2. Hegi ME, Diserens AC, Gorlia T, et al. MGMT gene silencing and benefit from temozolomide in glioblastoma. $N$ Engl J Med. 2005;352:997-1003.

3. Verhaak RG, Hoadley KA, Purdom E, et al. Integrated genomic analysis identifies clinically relevant subtypes of glioblastoma characterized by abnormalities in PDGFRA, IDH1, EGFR, and NF1. Cancer Cell. 2010;17:98-110.

4. Carro MS, Lim WK, Alvarez MJ, et al. The transcriptional network for mesenchymal transformation of brain tumours. Nature. 2010;463:318-325

5. Long DM. Capillary ultrastructure and the blood-brain barrier in human malignant brain tumors. J Neurosurg. 1970;32:127-144.

6. McClelland S 3rd, Long DM. Genesis of the use of corticosteroids in the treatment and prevention of brain edema. Neurosurgery. 2008;62:965-967; discussion 967-8.

7. Zibera C, Gibelli N, Butti G, et al. Proliferative effect of dexamethasone on a human glioblastoma cell line (HU 197) is mediated by glucocorticoid receptors. Anticancer Res. 1992;12:1571-1574.

8. Kaup B, Schindler I, Knupfer H, et al. Time-dependent inhibition of glioblastoma cell proliferation by dexamethasone. J Neurooncol. 2001;51:105-110.

9. Heiss JD, Papavassiliou E, Merrill MJ, et al. Mechanism of dexamethasone suppression of brain tumor-associated vascular permeability in rats. Involvement of the glucocorticoid receptor and vascular permeability factor. J Clin Invest. 1996;98:1400-1408.

10. Giraud S, Bessette B, Boda C, et al. In vitro apoptotic induction of human glioblastoma cells by Fas ligand plus etoposide and in vivo antitumour activity of combined drugs in xenografted nude rats. Int J Oncol. 2007;30:273-281.

11. Lin YM, Jan HJ, Lee CC, et al. Dexamethasone reduced invasiveness of human malignant glioblastoma cells through a MAPK phosphatase-1 (MKP-1) dependent mechanism. Eur J Pharmacol. 2008;593:1-9.

12. Villeneuve J, Galarneau H, Beaudet MJ, et al. Reduced glioma growth following dexamethasone or anti-angiopoietin 2 treatment. Brain Pathol. 2008;18:401-414.

13. Wong ET, Lok E, Gautam S, et al. Dexamethasone exerts profound immunologic interference on treatment efficacy for recurrent glioblastoma. Br J Cancer. 2015;113:232-241.

14. Singh SK, Hawkins C, Clarke ID, et al. Identification of human brain tumour initiating cells. Nature. 2004;432:396-401.

15. Reya T, Morrison SJ, Clarke MF, et al. Stem cells, cancer, and cancer stem cells. Nature. 2001;414:105-111.

16. Hossain A, Gumin J, Gao F, et al. Mesenchymal stem cells isolated from human gliomas increase proliferation and maintain stemness of glioma stem cells through the IL-6/gp130/STAT3 pathway. Stem Cells. 2015;33:2400-2415.

17. Shannon S, Vaca C, Jia D, et al. Dexamethasone-mediated activation of fibronectin matrix assembly reduces dispersal of primary human glioblastoma cells. PLoS One. 2015;10:e0135951.

18. Campana D, Manabe A, Evans WE. Stroma-supported immunocytometric assay (SIA): a novel method for testing the sensitivity of acute lymphoblastic leukemia cells to cytotoxic drugs. Leukemia. 1993; 7:482-488.

19. Cheema TA, Kanai R, Kim GW, et al. Enhanced antitumor efficacy of low-dose Etoposide with oncolytic herpes simplex virus in human glioblastoma stem cell xenografts. Clin Cancer Res. 2011;17:7383-7393.

20. Cheadle C, Cho-Chung YS, Becker KG, et al. Application of z-score transformation to affymetrix data. Appl Bioinformatics. 2003;2: 209-217.

21. Fu J, Khaybullin R, Liang X, et al. Discovery of gene regulation pattern in lung cancer by gene expression profiling using human tissues. Genom Data. 2015;3:112-115.

22. Cheadle C, Vawter MP, Freed WJ, et al. Analysis of microarray data using Z score transformation. J Mol Diagn. 2003;5:73-81.

23. Moroz MA, Huang R, Kochetkov $\mathrm{T}$, et al. Comparison of corticotropin-releasing factor, dexamethasone, and temozolomide: treatment efficacy and toxicity in U87 and C6 intracranial gliomas. Clin Cancer Res. 2011;17:3282-3292.
24. Srinivasan S, Patric IR, Somasundaram K. A ten-microRNA expression signature predicts survival in glioblastoma. PLoS One. 2011;6:e17438.

25. Celiku O, Johnson S, Zhao S, et al. Visualizing molecular profiles of glioblastoma with GBM-BioDP. PLoS One. 2014;9:e101239.

26. Lamb J, Crawford ED, Peck D, et al. The Connectivity Map: using gene-expression signatures to connect small molecules, genes, and disease. Science. 2006;313:1929-1935.

27. Wei G, Twomey D, Lamb J, et al. Gene expression-based chemical genomics identifies rapamycin as a modulator of MCL1 and glucocorticoid resistance. Cancer Cell. 2006;10:331-342.

28. Hieronymus H, Lamb J, Ross KN, et al. Gene expression signaturebased chemical genomic prediction identifies a novel class of HSP90 pathway modulators. Cancer Cell. 2006;10:321-330.

29. Subramanian A, Tamayo P, Mootha VK, et al. Gene set enrichment analysis: a knowledge-based approach for interpreting genomewide expression profiles. Proc Natl Acad Sci USA. 2005;102:15545-15550.

30. Lee JK, Joo KM, Lee J, et al. Targeting the epithelial to mesenchymal transition in glioblastoma: the emerging role of MET signaling. Onco Targets Ther. 2014;7:1933-1944.

31. Wen PY, Schiff D, Cloughesy TF, et al. A phase II study evaluating the efficacy and safety of AMG 102 (rilotumumab) in patients with recurrent glioblastoma. Neuro Oncol. 2011;13:437-446.

32. Bunn HF, Poyton RO. Oxygen sensing and molecular adaptation to hypoxia. Physiol Rev. 1996;76:839-885.

33. Maxwell PH, Wiesener MS, Chang GW, et al. The tumour suppressor protein VHL targets hypoxia-inducible factors for oxygen-dependent proteolysis. Nature. 1999;399:271-275.

34. Dreyfuss JM, Johnson MD, Park PJ. Meta-analysis of glioblastoma multiforme versus anaplastic astrocytoma identifies robust gene markers. Mol Cancer. 2009;8:71.

35. Zhang SD, Leung KL, McCrudden CM, et al. The prognostic significance of combining VEGFA, FLT1 and KDR mRNA expressions in brain tumors. J Cancer. 2015;6:812-818.

36. Kreisl TN, Kim L, Moore K, et al. Phase II trial of single-agent bevacizumab followed by bevacizumab plus irinotecan at tumor progression in recurrent glioblastoma. J Clin Oncol. 2009;27: $740-745$.

37. Friedman HS, Prados MD, Wen PY, et al. Bevacizumab alone and in combination with irinotecan in recurrent glioblastoma. J Clin Oncol. 2009;27:4733-4740.

38. Liu C, Zhang Y, Zhang K, et al. Expression of estrogen receptors, androgen receptor and steroid receptor coactivator-3 is negatively correlated to the differentiation of astrocytic tumors. Cancer Epidemiol. 2014;38:291-297.

39. Harmalkar M, Upraity S, Kazi S, et al. Tamoxifen-induced cell death of malignant glioma cells is brought about by oxidativestress-mediated alterations in the expression of BCL2 family members and is enhanced on miR-21 inhibition. J Mol Neurosci. 2015;57:197-202.

40. He W, Liu R, Yang SH, et al. Chemotherapeutic effect of tamoxifen on temozolomide-resistant gliomas. Anticancer Drugs. 2015;26: 293-300.

41. Chowdhury UR, Samant RS, Fodstad O, et al. Emerging role of nuclear protein 1 (NUPR1) in cancer biology. Cancer Metastasis Rev. 2009;28:225-232.

42. Jiang WG, Davies G, Martin TA, et al. Com-1/p8 acts as a putative tumour suppressor in prostate cancer. Int $J$ Mol Med. 2006;18: 981-986.

43. Bastien JI, McNeill KA, Fine HA. Molecular characterizations of glioblastoma, targeted therapy, and clinical results to date. Cancer. 2015;121:502-516.

44. Furnari FB, Fenton T, Bachoo RM, et al. Malignant astrocytic glioma: genetics, biology, and paths to treatment. Genes Dev. 2007;21:2683-2710.

45. Chai KM, Wang CY, Liaw HJ, et al. Downregulation of BRCA1BRCA2-containing complex subunit 3 sensitizes glioma cells to temozolomide. Oncotarget. 2014;5:10901-10915.

46. Roth P, Wick W, Weller M. Steroids in neurooncology: actions, indications, side-effects. Curr Opin Neurol. 2010;23: 597-602. 